\title{
Sestamibi Positive Vs Negative Scan In Primary Hyperparathyroidism; A Clinical Dilemma
}

\author{
Hosne Ara Rahman, Jasmine Ara Haque, Samira Sharmin \\ Institute of Nuclear Medicine and Allied Sciences, Mitford, Dhaka. \\ For correspondence: Dr. Hosne Ara Rahman, Principal Medical Officer, Institute of Nuclear Medicine and \\ Allied Science, Sir Salimullah Medical College Campus, Mitford, Dhaka. E-mail : dr_anunmc07@yahoo.com
}

\begin{abstract}
Objectives: In primary hyperthyroidism Tc-99m Sestamibi (MIBI) scanning is commonly used for localization of abnormal parathyroid gland and the reported sensitivity is very high. However, false negative scan remain a problem. We examined whether serum calcium and parathormone (PTH) level have any impact in the sensitivity of MIBI scan.

Study design: A retrospective review of 55 patients with primary hyperparathyroidism who underwent MIBI scan from January 2012 to December 2014 were included in this study. All patients underwent parathyroid surgery followed by histopathological confirmation.

Results: In total, 55 patients were studied. Mean age was 41.3 \pm 19.8 years (range 21-63 years). MIBI scan was true positive in 37 cases and false negative in 18 cases. The sensitivity of MIBI scan was $67.2 \%$. Mean serum calcium level was $12.3 \mathrm{mg} / \mathrm{dL}$. More than $62.2 \%$ of patients with calcium level greater than $12.3 \mathrm{mg} / \mathrm{dL}$ had a positive scan as compared with $37.8 \%$ of those with lesser value $(\mathbf{P}<0.05)$. Similarly a serum PTH level greater than $316 \pm$ $139 \mathrm{pg} / \mathrm{mL}$ correlated with positive scans in $78.4 \%$ as opposed to $21.6 \%$ in those with lower levels $(P<0$.01).
\end{abstract}

Conclusion: Lower calcium and PTH level significantly correlate with reduced sensitivity of MIBI scan. Although we did not find the best cut-off level of serum calcium and PTH level that can predict a positive scan.

Key words: Primary hyperparathyroidism, Sestamibi scan, Hypercalcemia, Parathormone (PTH).

\section{INTRODUCTION}

In recent years primary hyperparathyroidism has been diagnosed with increasing frequency due to increase awareness and advances in laboratory techniques. With the increase of biochemical screening even asymptomatic primary hyperparathyroidism is diagnosed. Of all available imaging modalities Tc-99m sestamibi scanning has become the modality of choice as compared to other modalities such as ultrasonography, CT and MRI.
The reported sensitivity of MIBI scan ranges from $70-85 \%$, which increases to $90-95 \%$ especially when imaging, is optimized with SPECT and SPECT/CT (1). In spite of that a substantial number of patients with elevated PTH level may have false negative sestamibi scan. In these cases, no adenoma/hyperplasia is visualized on the scan although the patient may have parathyroid pathology leading to diagnostic dilemma. The aim of this review study is to see whether serum calcium and PTH level have any impact in the sensitivity of MIBI scan.

\section{MATERIALS AND METHODS}

A retrospective review of 55 patients with primary hyperparathyroidism referred to our department for Tc-99m sestamibi parathyroid scanning between January 2012 to December 2014 were included in this study. Diagnostic criteria for primary hyperparathyroidism were based on high PTH level associated with hypercalcemia. In this study normal reference range for serum PTH was 9-80 $\mathrm{pg} / \mathrm{mL}$ and serum calcium was $8.3-10.1 \mathrm{mg} / \mathrm{dL}$. The dual - phase method of parathyroid imaging was conducted using standard protocol. All patients received $20 \mathrm{mCi}$ Tc-99m sestamibi intravenously. Early phase (20-minutes post injection) and delayed phase (2-hour post injection) SPECT images were acquired on a dual head SPECT/CT system (Symbia, Siemens). The images were acquired into a $128 \times 128$ matrix with $30 \mathrm{sec}$ per step having 60 steps over a fall $360^{\circ}$ orbit. In delayed view a low dose CT was also performed covering the area of SPECT acquisition. 
Transverse images were reconstructed in coronal and sagittal views. Sestamibi scan were designated as either 'positive' or 'negative'. A positive scan was defined when an area of relatively increased radiotracer uptake in early phase persisted and became more prominent in delayed phase because of slower washout of radiotracer from parathyroid than from thyroid gland. Conversely negative scan was considered when delayed images show no unusual activity in the neck region. The results were correlated with serum calcium and PTH level. All patients underwent surgery followed by histopathological confirmation.

Statistical analysis was performed using SPSS software version 15. Effects of variable on the result of MIBI scan were studied using chi-square and regression analysis.

$\mathrm{P}$ value $<0.05$ was considered statistically significant.

\section{RESULTS}

A total 55 patients with hypercalcaemia and high serum PTH level were studied. Mean age was 41.3 \pm 19.8 years (range 21-63 years). Female were predominant than male which was $31(56.4 \%)$ and $24(43.6 \%)$ respectively. MIBI scan was true positive in $67.2 \%(\mathrm{n}=37)$ cases and false negative in $32.8 \%(\mathrm{n}=18)$ cases. The overall sensitivity of MIBI scan was $67.2 \%$. Mean PTH level was $316 \pm$ $139 \mathrm{pg} / \mathrm{mL}$. Among the 37 patients with positive scan $29(78.3 \%)$ had serum PTH level above the mean value whereas, rest eight $(21.7 \%)$ had below the mean value. Meanwhile among 18 patients with negative scan 7 (38.8\%) had a PTH level above the mean value and $11(61.2 \%)$ had below the mean level $(\mathrm{P}<0.01)$. Mean PTH level in true positive and in false negative scan group was $412 \pm 259 \mathrm{pg} / \mathrm{mL}$ and $215 \pm 137 \mathrm{pg} / \mathrm{mL}$ respectively. Similarly, among the patients having positive scan $62.2 \%(n=23)$ had serum calcium level greater than $12.3 \mathrm{mg} / \mathrm{dL}$ as opposed to $37.8 \%(n=14)$ in those with lower level. Among 18 false negative cases, five $(27.7 \%)$ had below and $13(72.3 \%)$ had above the mean value of calcium $(\mathrm{p}<0.05)$.

\section{DISCUSSION}

MIBI scan is the current imaging modality of choice for localization of abnormal parathyroid gland. A normal parathyroid gland does not take up sestamibi. The exact mechanism of its selective uptake in abnormal parathyroid gland remains debatable (1). High mitochondrial activity is considered to be the major component of tracer uptake by parathyroid tissue in-patient with high PTH level. Moreover, several factors that have been reported to be associated with sestamibi imaging are size of gland, multiglandular disease, histopathological variants, serum calcium level, PTH level, vitamin D level and use of calcium channel blockers (2). The overall sensitivity of MIBI scan documented in several studies ranges from 70 to $85 \%$. In this study the sensitivity is $67.2 \%$, which is lower than the reported value. According to the study result, higher level of PTH is significantly related with a true positive scan $(\mathrm{P}<0.01)$. Mean PTH level in patients with true positive scan is $412 \pm 259 \mathrm{pg} / \mathrm{mL}$ and $215 \pm 137$ $\mathrm{pg} / \mathrm{mL}$ in patients with false negative scan which gives an impression of correlation between sensitivity of MIBI scan and PTH level. Our findings are consistent with Siegel et al. who reported a correlation between the sensitivity of parathyroid scintigraphy and PTH level. In their retrospective review mean PTH level were 367 $\mathrm{pg} / \mathrm{mL}$ in true positive scan group and $148 \mathrm{pg} / \mathrm{mL}$ in false negative scan group (3). Similarly Parikshak et al. showed the same finding where low PTH level and sensitivity of the MIBI scan are inversely related. Comparing the parathormone level their mean PTH level was $158 \mathrm{pg} / \mathrm{mL}$ whereas in our study it is higher (4).

Regarding the calcium level, a review of literature focused on technical and biological factors affecting MIBI scan showed that the plasma calcium greater than $11.3 \mathrm{mg} / \mathrm{dL}$ had a positive scan compared to those with lesser value. 


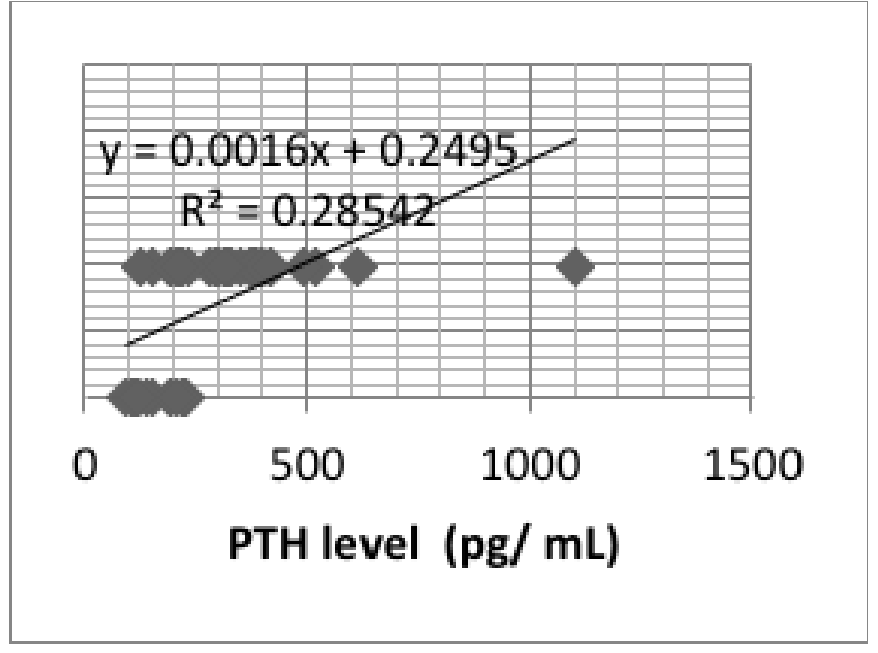

Figure 1: Scatter plot showing the degree of correlation between the serum PTH level ( $x$-axis) and MIBI scan finding (y-axis).

The degree of hypercalcaemia in primary hyperparathyroidism seems to be closely related to defective regulation of PTH secretion, metabolic rate and elevated membrane potential rather than increased mass of parathyroid tissue (5). Indeed, in some patient hypercalcaemia may be surprisingly increased despite only modest glandular enlargement. Therefore, serum calcium level in primary hyperparathyroidism may play a key role in modifying MIBI kinetics by influencing the membrane potential. In this study mean serum calcium level is $12.3 \mathrm{mg} / \mathrm{dL} .37 .8 \%$ of true positive scan had a calcium level below the mean value whereas, $62.2 \%$ had above the mean value. On the other hand in false negative cases $72.3 \%$ had a calcium level below the mean value and $27.7 \%$ had above the mean value. These findings have similarities with Saanislaw et al. study where serum calcium level was significantly higher in positive scan cases $(\mathrm{p}<0.04)(6)$.

Hypercalcaemia accompanied by elevated PTH level is diagnostic of hyperparathyroidism. However, a cautionary note is that during the review we found a minority of scans positive patients who had serum calcium levels in upper limit of normal reference range. This is called normocalcemic hyperparathyroidism.

Table 1: Correlation of sestamibi scans result (true positive Vs false negative) with mean calcium and PTH level.

\begin{tabular}{|l|c|c|}
\hline \multicolumn{1}{|c|}{ Scan designation } & $\begin{array}{c}\text { Mean calcium } \\
\mathbf{( m g / d L )}\end{array}$ & $\begin{array}{c}\text { Mean } \\
\text { PTH }(\mathbf{p g} / \mathbf{m L})\end{array}$ \\
\hline True positive $(\mathrm{n}=37)$ & $13.2 \pm 1.9$ & $412 \pm 259$ \\
False negative $(\mathrm{n}=18)$ & $10.9 \pm 0.91$ & $215 \pm 137$ \\
Significance & $\mathrm{p}<0.05$ & $\mathrm{p}<0.01$ \\
\hline
\end{tabular}

We did not include these cases in our review but, it should be kept in mind that the condition is an indication of early diagnosis of the disease. According to Westerdahl and Bergenflez study high glandular weight and high PTH level were important factors for delectability of adenoma (7). In contrary there are literatures where the investigators showed no significant correlation between MIBI uptake and serum calcium and PTH level (8).

This study also showed that some other biochemical profile like serum albumin, magnesium and inorganic phosphate were low in patients with a positive scan though the relation was not so significant. Among other influencing factors size of the gland is thought to be an important one to affect the positivity of the scan. In a report by Richard et al., an adenoma weight $>600 \mathrm{mg}$ and oxyphil content $>20 \%$ increased the rate of obtaining a positive scan by 10 and 4 fold respectively (9). In this study neither weight of the gland nor the cellular type of the gland were included.

The limitation of the study was small study population and ignoring other influencing factors. We only emphasized on the importance of PTH and calcium level in predicting the result of the MIBI scan. In our small-scale study we were unable to identify a figure at which we can fully predict that any given abnormal parathyroid gland will yield a positive result. 
Further large cohorts are needed for a definite consensus to find out the best cut-off level of biochemical parameter that can predict a positive scan.

\section{CONCLUSION}

It was important to note that, diagnosis of primary hyperparathyroidism was made on clinical and biochemical data. There was no definite lower limit of serum PTH and calcium level which can predict a positive scan. So, by understanding the limitations in each modality clinician should take the therapeutic decision.

\section{REFERENCES}

1. Mshelia DS, Hatutale AN, Mokgoro NP, Nchabaleng ME, Buscombe JR, Sathekge MM. Correlation between serum Calcium level and dual phase $99 \mathrm{mTc}$-sestamibi parathyroid scintigraphy in primary hyperparathyroidism. Clinical physiology and functional imaging 2011; 32(11):19-24.

2. Denhan DW, Normens J. Cost-effectiveness of preoperative sestamibi scan for primary hyperparathyroidism dependent solely on surgeon's choice of operative procedure. J Am Coll Surg 1998; 186:293-314.

3. Siegel A, Alvarado M ,Barsth Jr, Brady M, Lewis J. Parameter in prediction of the nuclear sensitivity of parathyroid scan. Clin Nucl Med 2006; 31:69699.
4. Parikshak M, Calciumstillo ED, Conrad MF, Talpos GB. Impact of hypercalcemia and PTH level in the sensitivity of preoperative sestamibi scan for primary hyperparathyroidism. Am Surg 2003; 69:393-98.

5. Kannan S, Milas M, Neumann D, Parikh RT, Sipcerstein A, Licata A. Parathyroid nuclear scan: A focused review on technical and biological factors affecting its outcome . Clin Miner Bone Metab 2014; 11(1): 25-30.

6. Stawick SP, Chaar ME, Baillie DR, Jaik NP, Estrada FP. Correlation between biochemical testing pathology findings and preoperative sestamibi scans: a retrospective study of the minimally invasive radio guided parathyroidectomy approach. Nucl Med Review 2007;1 0:82-86.

7. Westerdahl J, Bergenflez A. Sestamibi scan directed parathyroid surgery: potentially high failure rate without measurement of intraoperative parathyroid hormone. World $\mathrm{J}$ Surg 2004: 1132-8.

8. Cermik TF, Puyan FO, Sezer A, Firat MF, Berkarda S. Relation between Tc-99m sestamibi uptake and biological factors in hyperparathyroidism. Ann Nucl Med 2005; 19: 387-92.

9. Westreich R, Brandwein M, Mechanick JL,Bergman DA, Urken ML. Preoperative parathyroid localization: correlating falsenegative technetium $99 \mathrm{~m}$ sestamibi scan with parathyroid disease. Laryngoscope 2002; 113:567-572. 